\title{
Epidemiology of diabetic foot disease and diabetes-related lower-extremity amputation in Australia: a systematic review protocol
}

\author{
Jaap J. van Netten ${ }^{1,2,3^{*}}$, Mendel Baba ${ }^{5}$ and Peter A. Lazzarini ${ }^{1,2,3,4}$
}

\begin{abstract}
Background: Diabetic foot disease is associated with major morbidity, mortality, costs, and reduction of a person's quality of life. Investigating the epidemiology of diabetic foot disease is the backbone of diabetic foot research and clinical practice, yet the full burden of diabetic foot disease in Australia is unknown. This study aims to describe the protocol for a systematic review of the epidemiology of diabetic foot disease and diabetes-related lower-extremity amputation in Australia.
\end{abstract}

Methods_search: The systematic review will be performed according to the Preferred Reporting Items for Systematic Review and Meta-Analyses guidelines. PubMed and EMBASE will be searched for publications in any language and without restrictions to date. Two independent investigators will screen publications for eligibility, with publications reporting Australian population-based incidence or prevalence of diabetic foot disease or diabetes-related lowerextremity amputation to be included. Additionally, a forward literature search will be performed in Google Scholar, and a grey literature search will be performed to identify government publications.

Methods_assessment: Quality assessment will be performed using customised checklists. The summary statistic used for each study will be an incidence or prevalence proportion of diabetic foot disease or diabetes-related lower-extremity amputation. The standard error for each proportion will be calculated. A meta-analysis will be performed when three or more publications of adequate quality, reporting on similar outcomes and in similar populations, are identified.

Discussion: The results of this systematic review can be used to adequately inform stakeholders in the field of diabetic foot disease on the extent of the problem in incidence and prevalence of diabetic foot disease in Australia, and to help guide appropriate use of resources to reduce the burden of this disease.

Systematic review registration: PROSPERO CRD42016050740

Keywords: Diabetes mellitus, Diabetic foot disease, Foot ulcer, Amputation, Epidemiology, Incidence, Prevalence, Australia

\section{Background}

Diabetic foot disease is a complication of diabetes associated with major morbidity, mortality, costs, and reduced quality of life [1-4]. Diabetic foot disease typically presents as ulcers, infection, and Charcot foot in the presence of peripheral neuropathy or peripheral arterial disease in

\footnotetext{
* Correspondence: jaap.vannetten@qut.edu.au

${ }^{1}$ School of Clinical Sciences, Queensland University of Technology, Brisbane, QLD, Australia

${ }^{2}$ Diabetic Foot Australia, West End, QLD 4101, Australia

Full list of author information is available at the end of the article
}

people with diabetes [5], and is the most important precursor for lower-extremity amputations [6]. Global prevalence of diabetic foot disease is estimated around 6\% [7], whilst diabetes-related lower-extremity amputation incidence shows great global variability [8].

Investigating the epidemiology of a disease is the backbone of both research and clinical practice. Quantification of the problem is required to direct service provision, enable efforts for improvement, benchmark disease outcomes, and act as baseline to measure change. Australia has been reported to have one of the lowest rates of 
diabetic foot disease [7], yet one of the highest rates of diabetes-related amputation $[9,10]$. However, these rates have never been systematically investigated. Therefore, the full burden of diabetic foot disease in Australia is unknown.

Whilst Australia is similar to other developed nations with regard to its ageing and urban population [11], it has many unique characteristics to other nations. For instance, Australia's rural population is spread over much larger geographical distances and therefore access to health system services can prove more difficult compared to other developed nations. Furthermore, the indigenous population with diabetes in Australia has been reported to be 38 times more likely to undergo amputation than the non-indigenous (predominantly Caucasian) population [12]. These factors make Australia relatively unique compared to other developed nations, particularly European nations with much smaller geographical differences, and with the non-Caucasian populations presenting with lower risks of amputation compared to the Caucasian population [13].

All these factors warrant the need for a study investigating the burden of this disease in Australia. This study aims to systematically review the epidemiology of diabetic foot disease and diabetes-related lower-extremity amputation in Australia.

\section{Methods}

The systematic review will be performed according to the Preferred Reporting Items for Systematic Review and Meta-Analyses (PRISMA) guidelines, with this protocol written according to the PRISMA-P checklist (see Additional file 1: Appendix 1) [14, 15]. The systematic review has been prospectively registered in the PROSPERO database for systematic reviews (CRD42016050740). Protocol amendments will be documented in PROSPERO.

\section{Search strategy}

The following databases will be searched: PubMed and Excerpta Medica Database (EMBASE) via Ovid SP and will include publications in any language and without restrictions in date. Before performing the search, a validation set of approximately 10 publications was created and tested. Each publication in the set needed to be identified in the search. The validation set was created by including key publications known to the authors that fit the scope of this systematic review. The final search string is shown in Table 1.

\section{Data management}

Preliminary searches resulted in 155 records and 20 potentially eligible publications. Records and data can therefore be managed using Microsoft Excel (Microsoft Corporation, Redmond, WA, USA). All records will be imported from PubMed and EMBASE into an Excel file.
This file will be used for study selection. Keeping the master copy and all management of this file is the responsibility of the first author. The Statistical Package for the Social Sciences (IBM Corporation, Armonk, NY, USA) will be used for data analysis, with data imported by the first author.

\section{Study selection}

Title and abstracts will be screened by two independent investigators ( JvVN and $\mathrm{MB}$ ) for inclusion in the full-text assessment. Criterion for inclusion for full-text assessment is any mention of epidemiological data on diabetic foot disease or lower-extremity amputation in an Australian setting. A publication identified for inclusion by either one of the investigators will be included for full-text assessment. Cohen's kappa will be calculated for screening agreement by the two investigators [16].

Included full-text publications will be assessed by two independent investigators (JJvN and $\mathrm{MB}$ ) for eligibility using the inclusion and exclusion criteria. Inclusion criteria will be based on publications reporting Australian population-based incidence or prevalence of diabetic foot disease or diabetes-related lower-extremity amputation. Exclusion criteria will be based on publications reporting data from a single centre or selected centres (unless general- or diabetes-population information for the selected centres is provided or if data is adjusted or compared to the overall Australian population demographics); publications reporting lower-extremity amputation incidence or prevalence without differentiating between people with and without diabetes; and publications not reporting original data. No restrictions are placed on study duration, study period, or date of publication. Disagreements between investigators will be resolved with discussion until consensus is reached, with a third investigator to be involved if needed.

Diabetic foot disease will be defined as "infection, ulceration or destruction of tissues of the foot associated with neuropathy and/or peripheral artery disease in the lower extremity of people with diabetes" [17]. Diabetesrelated lower-extremity amputation will be defined as "resection of a segment of the lower-extremity through a bone or joint" in a person with diabetes [17]. This will be further differentiated as major amputation (any resection proximal of the ankle) and minor amputation (any resection through or distal of the articulation of the ankle) [17]. However, we are mindful that different definitions may have been used in different studies and we will also include (and report) other definitions if used.

The denominator population will be defined as the general or diabetes population reported for a specified geographical area, with key characteristics (age and gender) to be reported for this population in the publication. Publications reporting in-hospital epidemiology of 
Table 1 Search strings for PubMed and EMBASE

\begin{tabular}{|c|c|c|c|}
\hline & PubMed & & EMBASE \\
\hline$\#$ & Diabetic foot disease string & $\#$ & Diabetic foot disease string \\
\hline 1 & (“Ulcer"[Mesh] OR ulcer*[tw]) & 1 & 'ulcer'/exp \\
\hline 2 & $\begin{array}{l}\text { ("Foot"[Mesh] OR foot[tw] } \\
\text { OR feet[tw]) }\end{array}$ & 2 & ulcer:ab,ti \\
\hline 3 & "Foot Ulcer"[Mesh] & 3 & \#1 OR \#2 \\
\hline 4 & $\begin{array}{l}\text { ("Foot Diseases"[Mesh] OR foot } \\
\text { problem*[tw] OR foot disease*[tw]) }\end{array}$ & 4 & 'foot'/exp \\
\hline 5 & diabet*$^{*}[\mathrm{tw}]$ & 5 & (foot or feet):ab,ti \\
\hline 6 & \#1 AND \#2 AND \#5 & 6 & \#4 OR \#5 \\
\hline 7 & \#3 AND \#5 & 7 & \#3 AND \#6 \\
\hline 8 & \#4 AND \#5 & 8 & 'foot ulcer'/exp \\
\hline 9 & diabetic foot[tw] & 9 & 'foot disease'/exp \\
\hline 10 & diabetic feet[tw] & 10 & $\begin{array}{l}\text { (foot disease* or } \\
\text { foot problem*):ab,ti }\end{array}$ \\
\hline \multirow[t]{8}{*}{11} & \#6 OR \#7 OR \#8 OR \#9 OR \#10 & 11 & diabet*ab,ti $^{*}$ \\
\hline & & 12 & \#7 AND \#11 \\
\hline & & 13 & \#8 AND \#11 \\
\hline & & 14 & \#9 AND \#11 \\
\hline & & 15 & \#10 AND \#11 \\
\hline & & 16 & 'diabetic foot'/exp \\
\hline & & 17 & $\begin{array}{l}\# 12 \text { OR \#13 OR \#14 } \\
\text { OR \#15 OR \#16 }\end{array}$ \\
\hline & Amputation string & & Amputation string \\
\hline 12 & Amput* $[\mathrm{tw}]$ & 18 & 'amput*/exp \\
\hline 13 & Diabet* $^{*}[\mathrm{tw}]$ & 19 & diabet $^{*}: a b, t i$ \\
\hline \multirow[t]{2}{*}{14} & \#12 AND \#13 & 20 & \#18 AND \#19 \\
\hline & Outcomes string & & Outcomes string \\
\hline 15 & Incidence & 21 & Incidence/exp \\
\hline 16 & Prevalence & 22 & Prevalence/exp \\
\hline 17 & (\#15 OR \#16) & 23 & \#21 OR \#22 \\
\hline 18 & Austral* & 24 & Austral*/exp \\
\hline \multirow[t]{2}{*}{19} & \#17 AND \#18 & 25 & \#23 AND \#24 \\
\hline & Final search & & Final search \\
\hline 20 & \#11 OR \#14 & 26 & \#17 OR \#20 \\
\hline 21 & \#19 AND \#20 & 27 & \#25 AND \#26 \\
\hline
\end{tabular}

diabetic foot disease or diabetes-related lower-extremity amputation will be included, when data from the complete hospital population is given, and provided more than one hospital is included in the publication. Common risk factors for diabetic foot disease may also be used to further specify the "at-risk population" in the denominator (when available in a publication), and these include peripheral neuropathy, peripheral artery disease, and foot deformities $[17,18]$.

After completion of selection of the final included publications, a forward literature search will be performed via
Google Scholar (https://scholar.google.com.au). This may help to identify early online publications that have not been indexed (since Google Scholar does not rely on indexation before publications are included in their database), and it may capture some grey literature as well (such as dissertations and conference proceedings). One author will assess the publications found in the forward literature search. All publications identified for inclusion by this author will be screened for eligibility as per the above study selection procedure of full-text publications.

\section{Grey literature search}

As the authors are aware of government publications on the primary outcomes of this systematic review, a search to identify those publications and other grey literature meeting the inclusion and exclusion criteria will be undertaken. This search will consist of the following steps, performed by one author:

-Identifying and downloading the known Australian state and federal government publications meeting the inclusion and exclusion criteria

-Typing the title of the government publications in an incognito window of Google (www.google.com.au) and screening the first 10 pages of results by one investigator -Contacting the author of the government publications to inquire about any further reports, either following the publication found or additional publications -Contacting five external Australian content experts in the field to ask for known grey literature reporting diabetic foot disease epidemiology

As both EMBASE and Google Scholar include conference presentations and dissertations, the searches in those databases described elsewhere in this manuscript can also be regarded as covering part of the grey literature search.

All publications identified for inclusion in the grey literature search will be screened for eligibility as per the above study selection procedure of full-text publications.

\section{Data collection}

Data from each publication will be extracted by one investigator, after which a second investigator will check the extracted data. Investigators will not extract data from an article of which they were (co-)author to prevent potential bias or conflict of interest. The primary outcome is the incidence or prevalence of diabetic foot disease and diabetes-related lower-extremity amputation. Data extracted will include setting, period, study design, use of prevalence or incidence, number of general or diabetes population, key characteristics (age, sex, foot disease risk factors, other) within the population, definition of diabetic foot disease or amputation, classification 
of diabetic foot disease or amputation level, other characteristics of diabetic foot disease or amputation, and if the diabetic foot disease or amputation is the first ever incidence or a recurrence. Authors will be contacted if data to be extracted are unclear from the original publication.

\section{Quality assessment}

As diabetic foot disease and amputation pose specific requirements on study reporting, no specific quality assessment instrument is suited to the data and research aim of this review. Therefore, a customised approach will be followed. In line with conclusions from a recent systematic review on tools for assessing the quality and susceptibility to bias in observational studies, a transparent checklist approach has been chosen, concentrating on the few-principal-potential sources of bias identified from this systematic review [19]. The customised checklist developed to assess selection and information bias by the investigators is based on checklists used by other systematic reviews on population-based studies investigating mortality after amputation [20] and diabetic foot disease [21], which in turn were based broadly on the Meta-analysis Of Observational Studies in Epidemiology guidelines [22] and the Newcastle-Ottawa scale [23]. Our checklist aims to assess selection and information bias.

Five criteria will be assessed for each included publication by two independent investigators (Table 2). Item $\mathrm{C}$ is outcome specific and is scored twice when both primary outcomes are included. Each criterion is scored with a "+", "?", or "-". The criteria chosen are based primarily on the quality of the reporting of the geographical area, diabetic foot disease or amputation numerator, population denominator, information flow, and study design. These criteria are all known to be important aspects of bias in diabetic foot disease research [21, 24]. Disagreements will be resolved by discussion until consensus is reached, with a third investigator to be involved if consensus is unable to be reached. Investigators will not assess the quality of an article of which they were (co-)author to prevent any conflict of interest.

Table 2 Customised checklist for risk of bias assessment

A Quality of description of included geographical area or data sources

+ Clear description of geographical area and participating centres within the area or data sources. For example, data are presented for the number, sex, and age distribution of the population. Where relevant, providing complete referencing details to original data collection procedures or sources is adequate

? Unclear or incomplete description of geographical area, its residents, and hospitals or data sources used

- No description of investigated area

B Quality of description of included population denominator

+ Clear description of inclusion and exclusion criteria is presented. Minimal criteria were considered sex, age, and (definition of) diabetes

? Unclear or incomplete description of inclusion and exclusion criteria

- No description of inclusion and exclusion criteria

C Quality of description of diabetic foot disease or amputation numerator Diabetic foot disease

Amputation

+ Clear description of reported diabetic foot disease characteristics in line with a published classification system using objective measurement methods

Clear description of the levels of amputation in line with international guidelines or codes, and a clear definition of whether amputations were the first, recurrent, or any in sequence

? Unclear or incomplete description of reported diabetic foot disease characteristics, or using subjective measurement methods

Unclear or incomplete description of levels of amputation in line with international guidelines or codes, or unclear or incomplete definition of sequence of amputation

- No description of diabetic foot disease characteristics No description of levels of amputation or sequence of amputation

D Quality of information flow

+ Information flow was complete for each stage of the study Are the number of individuals at each stage of study reported-including number potentially eligible, number included, and number completed to follow-up?

? Only national health statistics used, missing information for some stages or relevant time points

- Poor or no description given on each stage and missing all important information

E Quality of study design

+ Prospective study with diabetic foot disease or amputation as a predefined primary or secondary outcome

? Retrospective study with diabetic foot disease or amputation as a primary outcome

- Retrospective study with diabetic foot disease or amputation as a secondary outcome 
As grey literature is not peer-reviewed, an additional quality assessment of the grey literature will be performed, using the AACODS (Authority, Accuracy, Coverage, Objectivity, Date, Significance) checklist [25].

\section{Data synthesis}

The summary statistic used for each study will be an incidence (rate per population) or prevalence proportion (\%) of diabetic foot disease or diabetes-related lowerextremity amputation. The standard error for each proportion will be calculated.

A meta-analysis will be performed if three or more publications, with data collected within 10 years of each other, reporting on the same outcome and in a similar geographical area or with similar denominator population are found, providing that quality assessment items $\mathrm{A}, \mathrm{B}$, and $\mathrm{C}$ are scored with a "+". Meta-analysis will be used to calculate pooled incidence or prevalence estimates using a random-effects model. Random effects will be used to give an average estimate across heterogeneous studies weighted on total sample size. The $I^{2}$ test will be used to examine heterogeneity across studies included in meta-analysis.

No meta-bias or confidence in the cumulative evidence will be calculated or assessed. Availability of prospectively registered protocols of epidemiological studies is unlikely, and as such unpublished results to calculate publication bias cannot be investigated or estimated. Further, this systematic review does not concern intervention trials, rendering the Grading of Recommendations Assessment, Development and Evaluation system not applicable. The confidence in the cumulative evidence will be a descriptive analysis based on the separate quality assessment outcomes of the included studies.

\section{Discussion}

Epidemiological data are the backbone of research and clinical practice. However, epidemiological data are prone to various forms of bias and misinterpretation. The various intricacies of diabetic foot disease epidemiology, and their pros and cons for use in the assessment of the quality of care, have been frequently described [24, 26, 27]. However, a systematic review of the various sources of diabetic foot epidemiological data in which these intricacies are adequately taken into account and discussed is missing for the Australian situation. This may lead to the various stakeholders in this field (e.g., clinicians, researchers, politicians, and industry) misinterpreting the data, and either over- or understating the actual burden. The results of this systematic review can be used to adequately inform stakeholders in the field of diabetic foot disease and to help guide appropriate use of resources to reduce the burden of this debilitating disease.

\section{Additional file}

Additional file 1: Appendix 1. PRISMA-P 2015 Checklist. (PDF 199 kb)

Abbreviations

EMBASE: Excerpta Medica Database; PRISMA: Preferred Reporting Items for Systematic Review and Meta-Analyses

Acknowledgements

The authors would like to acknowledge the support of the Australian Government's Cooperative Research Centres Program.

\section{Funding}

This systematic review was commissioned by Diabetic Foot Australia. The contributions of the first author are supported by the Australian Government's Cooperative Research Centres Program. The Queensland University of Technology is the sponsor of this systematic review. None of them had a role in developing the protocol, which is the sole responsibility of the authors.

Availability of data and materials Not applicable.

\section{Authors' contributions}

JJVN is the guarantor. JJVN and PAL drafted the protocol. All authors contributed to the selection and data extraction criteria and the risk of bias assessment strategy. JJVN drafted the search strategy, and MB and PAL critically reviewed the search strategy. PAL developed the data synthesis strategy. All authors read, provided feedback, and approved the final protocol.

\section{Authors' information}

JJVN is scientific director of Diabetic Foot Australia. PAL is co-chair of Diabetic Foot Australia.

\section{Competing interests}

The authors declare that they have no competing interests.

Consent for publication

Not applicable.

Ethics approval and consent to participate

Not applicable.

\section{Publisher's Note}

Springer Nature remains neutral with regard to jurisdictional claims in published maps and institutional affiliations.

\section{Author details}

${ }^{1}$ School of Clinical Sciences, Queensland University of Technology, Brisbane, QLD, Australia. ${ }^{2}$ Diabetic Foot Australia, West End, QLD 4101, Australia. ${ }^{3}$ Wound Management Innovation Cooperative Research Centre, Brisbane, QLD, Australia. ${ }^{4}$ Department of Podiatry, Metro North Hospital \& Health Service, Queensland Health, Brisbane, QLD, Australia. ${ }^{5}$ Independent Consultant, Perth, Australia.

Received: 4 January 2017 Accepted: 28 April 2017

Published online: 18 May 2017

\section{References}

1. Boulton AJ, Vileikyte L, Ragnarson-Tennvall G, Apelqvist J. The global burden of diabetic foot disease. Lancet. 2005;366(9498):1719-24.

2. Kerr M, Rayman G, Jeffcoate WJ. Cost of diabetic foot disease to the National Health Service in England. Diabet Med. 2014;31(12):1498-504

3. Nabuurs-Franssen MH, Huijberts MS, Nieuwenhuijzen Kruseman AC, Willems J, Schaper NC. Health-related quality of life of diabetic foot ulcer patients and their caregivers. Diabetologia. 2005:48(9):1906-10.

4. Jupiter DC, Thorud JC, Buckley CJ, Shibuya N. The impact of foot ulceration and amputation on mortality in diabetic patients. I: From ulceration to death, a systematic review. Int Wound J. 2016;13(5):892-903. 
5. Schaper NC, Van Netten JJ, Apelqvist J, Lipsky BA, Bakker K, International Working Group on the Diabetic Foot. Prevention and management of foot problems in diabetes: a Summary Guidance for Daily Practice 2015, based on the IWGDF Guidance Documents. Diabetes Metab Res Rev. 2016;32 Suppl 1:7-15.

6. Martins-Mendes D, Monteiro-Soares M, Boyko EJ, Ribeiro M, Barata P, Lima J, et al. The independent contribution of diabetic foot ulcer on lower extremity amputation and mortality risk. J Diabetes Complications. 2014; 28(5):632-8.

7. Zhang P, Lu J, Jing Y, Tang S, Zhu D, Bi Y. Global epidemiology of diabetic foot ulceration: a systematic review and meta-analysis. Ann Med. 2016;1:1-21.

8. Moxey PW, Gogalniceanu P, Hinchliffe RJ, Loftus IM, Jones KJ, Thompson MM, et al. Lower extremity amputations-a review of global variability in incidence. Diabet Med. 2011;28(10):1144-53.

9. Lazzarini PA, Gurr JM, Rogers JR, Schox A, Bergin SM. Diabetes foot disease: the Cinderella of Australian diabetes management? J Foot Ankle Res. 2012; 5(1):24. $-1146-5-24$

10. Bureau of Health Information. Healthcare in Focus: 2010 - How NSW compares internationally. 2010

11. Australian Bureau for Statistics. Population by age and sex, Australia, States and Territories. 2016; Available at: http://www.abs.gov.au/ausstats/abs@.nsf/ 0/1CD2B1952AFC5E7ACA257298000F2E76?OpenDocument. Accessed 15 Dec 2016.

12. Norman PE, Schoen DE, Gurr JM, Kolybaba ML. High rates of amputation among Indigenous people in Western Australia. Med J Aust. 2010;192(7):421.

13. Holman N, Young RJ, Jeffcoate WJ. Variation in the recorded incidence of amputation of the lower limb in England. Diabetologia. 2012;55(7):1919-25.

14. Moher D, Liberati A, Tetzlaff J, Altman DG, PRISMA Group. Preferred reporting items for systematic reviews and meta-analyses: the PRISMA statement. J Clin Epidemiol. 2009;62(10):1006-12.

15. Moher D, Shamseer L, Clarke M, Ghersi D, Liberati A, Petticrew M, et al. Preferred reporting items for systematic review and meta-analysis protocols (PRISMA-P) 2015 statement. Syst Rev. 2015;4:1-4053-4-1.

16. Cohen J. A power primer. Psychol Bull. 1992;112(1):155-9.

17. Bakker K, Apelqvist J, Lipsky BA, Van Netten JJ, International Working Group on the Diabetic Foot. The 2015 IWGDF quidance documents on prevention and management of foot problems in diabetes: development of an evidencebased global consensus. Diabetes Metab Res Rev. 2016;32 Suppl 1:2-6.

18. Crawford F, Cezard G, Chappell FM, Murray GD, Price JF, Sheikh A, et al. A systematic review and individual patient data meta-analysis of prognostic factors for foot ulceration in people with diabetes: the international research collaboration for the prediction of diabetic foot ulcerations (PODUS). Health Technol Assess. 2015;19(57):1-210.

19. Sanderson S, Tatt ID, Higgins JP. Tools for assessing quality and susceptibility to bias in observational studies in epidemiology: a systematic review and annotated bibliography. Int J Epidemiol. 2007;36(3):666-76.

20. van Netten JJ, Fortington LV, Hinchliffe RJ, Hijmans JM. Early post-operative mortality after major lower limb amputation: a systematic review of population and regional based studies. Eur J Vasc Endovasc Surg. 2016;51(2):248-57.

21. Lazzarini PA, Hurn SE, Fernando ME, Jen SD, Kuys SS, Kamp MC, et al. Prevalence of foot disease and risk factors in general inpatient populations: a systematic review and meta-analysis. BMJ Open. 2015;5(11):e008544. -2015-008544.

22. Stroup DF, Berlin JA, Morton SC, Olkin I, Williamson GD, Rennie D, et al. Meta-analysis of observational studies in epidemiology: a proposal for reporting. Meta-analysis Of Observational Studies in Epidemiology (MOOSE) group. JAMA. 2000;283(15):2008-12.

23. Wells GA, Shea B, O'Connell D, Peterson J, Welch V, Losos M, et al. The Newcastle-Ottawa Scale (NOS) for assessing the quality of nonrandomised studies in meta-analyses. 2014; Available at: http://www.ohri.ca/programs/ clinical_epidemiology/oxford.asp. Accessed 01/2015.

24. Fortington LV, Geertzen JH, Bosmans JC, Dijkstra PU. Bias in amputation research; impact of subjects missed from a prospective study. PLoS One. 2012;7(8):e43629

25. Tyndall J. The AACODS checklist. 2010; Available at: http://dspace2.flinders edu.au/xmlui/bitstream/handle/2328/3326/AACODS_Checklist. pdf?sequence=4. Accessed 15 Dec 2015 .
26. Jeffcoate WJ, van Houtum WH. Amputation as a marker of the quality of foot care in diabetes. Diabetologia. 2004:47(12):2051-8.

27. Margolis DJ, Jeffcoate W. Epidemiology of foot ulceration and amputation: can global variation be explained? Med Clin North Am. 2013:97(5):791-805.

\section{Submit your next manuscript to BioMed Central and we will help you at every step:}

- We accept pre-submission inquiries

- Our selector tool helps you to find the most relevant journal

- We provide round the clock customer support

- Convenient online submission

- Thorough peer review

- Inclusion in PubMed and all major indexing services

- Maximum visibility for your research

Submit your manuscript at www.biomedcentral.com/submit
Biomed Central 